\title{
Sequence Analysis and Comparison of TCTP Proteins from Human Protozoan Parasites
}

\author{
Francisco Alejandro Lagunas-Rangel ${ }^{1}$ (i)
}

Received: 9 November 2021 / Accepted: 11 January 2022 / Published online: 9 February 2022

(c) The Author(s) 2022

\begin{abstract}
Purpose Translational controlled tumor protein (TCTP) is a functionally important protein in most eukaryotes because it participates in a wide variety of processes, the most representative being proliferation, differentiation, histamine release, cell death, protein synthesis and response to stress conditions. In the present work, we analyze the sequence, structure and phylogeny of TCTP orthologs in a group of human parasitic protozoan species.

Methods The complete sequences of TCTP orthologs in protozoan parasites were identified with the NCBI BLAST tool in the database of the EuPathDB Bioinformatics Resource Center. The sequences were aligned and important regions of the protein were identified, and later phylogenetic trees and 3D models were built with different bioinformatic tools.

Results Our results show evolutionarily and structurally conserved sites that could be exploited to create new therapeutic strategies given the increase in the number of strains resistant to current drugs.

Conclusion TCTP orthologs in protozoan parasites have been little studied but have been shown to be important in parasite growth, proliferation, reproduction, and response to changes in the environment. For all this, TCTP can be considered as a possible therapeutic target.
\end{abstract}

Keywords Histamine releasing factor $\cdot$ Phylogenetic analysis $\cdot$ Flexible loop $\cdot$ Dominant negative mutant

\section{Introduction}

Translational controlled tumor protein (TCTP), also called fortilin, P23 or histamine releasing factor (HRF), is a small multifunctional protein that is ubiquitous in eukaryotes [1]. This protein participates in a wide variety of key regulatory processes, such as control of proliferation and growth, pluripotency and differentiation, regulation of cell death, protein synthesis and degradation, the response to stress conditions and autophagy, microtubule stabilization, and histamine release, among others [2].

Particularly in human parasites, little is currently known about the functions and processes that involve TCTP orthologs, but some studies have shown that they collaborate in parasite growth, proliferation, reproduction and response to stress conditions, which is why this protein has been

Francisco Alejandro Lagunas-Rangel

francisco.lagunas@neuro.uu.se

1 Department of Surgical Sciences, Functional Pharmacology and Neuroscience, Uppsala University, Husargatan 3, BMC Box 593, 75124 Uppsala, Sweden suggested as a possible therapeutic target [3-5]. Toxoplasma gondii has been reported to require its TCTP ortholog for rapid growth and to maintain its intracellular development [3]. Moreover, TCTP has been shown to act as an antioxidant and heat shock protein with chaperone activity in some parasites [6-8] and can also interact and sequester antiparasitic drugs, thus contributing to the development of resistant strains [9-11]. TCTP has been reported to be present in the plasma of Plasmodium falciparum-infected patients at concentrations between 2.4 and $6.9 \mu \mathrm{g} / \mathrm{mL}$, indicating that these parasites secrete this protein [12]. In the same way, it has also been shown that other parasites also release this protein into the environment and it has been suggested that the TCTP protein secreted by the parasites could block the normal immune response by acting as a dominant negative mutant of human TCTP [4, 5, 13-15]. Notably, immunization of BALB/c mice with the purified recombinant TCTP protein from Plasmodium falciparum or Plasmodium yoelii reduced the parasitaemia generated during the early stages of Plasmodium yoelii or Plasmodium chabaudi infection and increased survival [16]. Likewise, a transient reduction in parasitemia was observed in $\mathrm{OF} 1$ mice infected with 
Trypanosoma brucei gambiense that were previously immunized with the parasite's TCTP protein [13]. The production of anti-TCTP antibodies in the immunized mice in both assays indicates that the protein is capable of inducing an adaptive immune response [13, 16]. Furthermore, TCTP of some parasites has been shown to play a role in the development of allergic inflammatory responses in infected patients $[14,17]$, stimulating the release of histamine from basophils and IL-8 from eosinophils [12]. For all this, it is thought that TCTP orthologs in parasites could act in two different areas, one involved in the regulation of host cytokine signaling and the other in the maintenance of parasite homeostasis and its response to conditions of stress [15].

The proposal of the present work was to analyze, from different human parasitic protozoan species, the sequence, structure and phylogeny of TCTP orthologs to identify notable characteristics that could be exploited in the development of new therapeutic strategies.

\section{Materials and Methods}

\section{Database Screening}

The orthologues of the human TCTP protein in protozoan parasites were found using the NCBI BLAST tool (with predetermined search parameters) in the database of the EuPathDB Bioinformatics Resource Center [18]. The complete sequences of the TCTP orthologs were compiled and included the following species: Homo sapiens (P13693), Cryptosporidium parvum (CPATCC_001190), Cryptosporidium hominis (Chro.30228), Toxoplasma gondii (TGME49_251680), Sarcocystis neurona (SN3_51680), Cyclospora cayetanensis (cyc_00965), Acanthamoeba castellanii (ACA1_069360 and ACA1_030760), Entamoeba histolytica (EHI_021360), Naegleria fowleri (FDP41_001128, NF0012290, FDP41_001927 and NF0009160), Giardia duodenalis (GL50803_6242 and DHA2_6242), Plasmodium falciparum (PF3D7_0511000), Trypanosoma brucei (Tb927.8.6750 and Tb927.8.6760), Trypanosoma cruzi (TcCLB.506207.50), Leishmania major (LmjF.24.1510 and LmjF.24.1500), Leishmania infantum (LINF_240020500 and LINF_240020600), Leishmania donovani (LdBPK_241560.1), Leishmania braziliensis (LbrM.24.1350), Leishmania mexicana (LmxM.24.1500a and LmxM.24.1500), and Trichomonas vaginalis (TVAG_180840).

\section{Sequence Analysis, Alignments, Phylogenetic Analysis, and Protein Modeling}

The sequences of the DDP4 proteins from all the organisms studied were analyzed using InterPro [19]. All the sequences obtained were aligned with the MEGA11 software [20] using the MUSCLE algorithm and then manually edited after visual inspection. Conservation calculations were performed with CLC Genomics Workbench 21 (QIAGEN Digital Insights). The aligned sequences were used to construct the phylogenetic trees using maximum likelihood and minimum evolution methods. The 3D models of the TCTP proteins were predicted with SWISS-MODEL [21], and illustrations were made using UCSF Chimera software [22].

\section{Results}

\section{Sequence Analysis}

After obtaining the complete sequences of the TCTP orthologs identified in the protozoan parasites, we performed an alignment (Supplementary Fig. 1) that showed an average identity of $30.9 \%$ and an overall similarity of $49.08 \%$ with respect to the human protein (Table 1). There was an overall identity of $38.19 \%$ among all proteins. The orthologist ACA1_069360 from A. castellanii was the one that had the greatest identity and similarity with the human protein. Protein sizes were around 151 and 229 amino acids (T. vaginalis and $N$. fowleri proteins, respectively) and the two protein fingerprints (TCTP1 and TCTP2) characteristic of this protein in all species were identified (Fig. 1). The TCPT1 fingerprint had $55.62 \%$ identity, while the TCTP2 fingerprint had $64.95 \%$. Considering the positions of our alignment, the most conserved residues were M107 (96\%), E123 (93\%), D127 (89\%), V186 (89\%), K202 (93\%), Y214 (89\%), K234 (93\%), F255 (100\%), G260 (96\%), P284 (93\%) and D291 (96\%), and L293 (89\%).

\section{Phylogenetic Analysis}

The sequence alignment of TCTP orthologs was used to build phylogenetic trees using two methods to have different perspectives, a character-based method such as maximum likelihood and a distance-based method such as minimum evolution (Fig. 2). Both methods constructed similar phylogenetic trees, placing the $N$. fowleri orthologs as the most ancestral proteins, while the orthologues of organisms in the Kinetoplastea class were the most evolutionarily advanced. Both trees separated TCTP orthologs from G. duodenalis and T. vaginalis, although both species belong to the Metamonada phylum. The T. vaginalis ortholog was closer to those of $N$. fowleri, while the $G$. duodenalis orthologs to those of the Kinetoplastea class. The orthologs of the species of the subphylum Apicomplexa and of the phylum Amoebozoa were placed in the middle of the proteins of $G$. duodenalis and T. vaginalis. The trees had differences when placing the human TCTP protein, the algorithm of 
Table 1 Identity and similarity of TCTP orthologues in protozoan parasites compared to human protein

\begin{tabular}{|c|c|c|c|c|}
\hline Species & UniProtKB $^{\mathrm{a}}$ & Size $^{b}$ & $\mathrm{I}^{\mathrm{c}}$ & $\mathrm{S}^{\mathrm{d}}$ \\
\hline $\begin{array}{l}\text { Cryptosporidium } \\
\text { parvum }\end{array}$ & CPATCC_001190 & 172 & 30.51 & 50.85 \\
\hline $\begin{array}{l}\text { Cryptosporidium } \\
\text { hominis }\end{array}$ & Chro.30228 & 172 & 30.51 & 50.85 \\
\hline Toxoplasma gondii & TGME49_251680 & 171 & 35.03 & 56.50 \\
\hline Sarcocystis neurona & SN3_51680 & 171 & 32.77 & 54.80 \\
\hline Cyclospora cayetanensis & cyc_00965 & 171 & 31.07 & 50.85 \\
\hline \multirow{2}{*}{$\begin{array}{l}\text { Acanthamoeba castel- } \\
\text { lanii }\end{array}$} & ACA1_069360 & 165 & 39.88 & 57.80 \\
\hline & ACA1_030760 & 191 & 35.75 & 52.85 \\
\hline Entamoeba histolytica & EHI_021360 & 170 & 36.42 & 57.23 \\
\hline \multirow[t]{4}{*}{ Naegleria fowleri } & FDP41_001128 & 229 & 21.19 & 33.05 \\
\hline & NF0012290 & 244 & 19.92 & 31.08 \\
\hline & FDP41_001927 & 171 & 27.78 & 46.67 \\
\hline & NF0009160 & 171 & 27.78 & 46.67 \\
\hline \multirow[t]{2}{*}{ Giardia duodenalis } & GL50803_6242 & 151 & 28.74 & 43.68 \\
\hline & DHA2_6242 & 151 & 28.74 & 43.68 \\
\hline Trichomonas vaginalis & TVAG_180840 & 151 & 26.16 & 39.53 \\
\hline Plasmodium falciparum & PF3D7_0511000 & 171 & 33.14 & 53.71 \\
\hline \multirow[t]{2}{*}{ Trypanosoma brucei } & Tb927.8.6750 & 170 & 32.96 & 53.63 \\
\hline & Tb927.8.6760 & 170 & 31.43 & 55.43 \\
\hline Trypanosoma cruzi & TcCLB.506207.50 & 170 & 30.43 & 51.09 \\
\hline \multirow[t]{2}{*}{ Leishmania major } & LmjF.24.1510 & 170 & 33.70 & 51.63 \\
\hline & LmjF.24.1500 & 170 & 33.70 & 51.63 \\
\hline \multirow[t]{2}{*}{ Leishmania infantum } & LINF_240020500 & 170 & 33.15 & 51.63 \\
\hline & LINF_240020600 & 170 & 33.15 & 51.63 \\
\hline Leishmania donovani & LdBPK_241560.1 & 170 & 33.15 & 51.63 \\
\hline Leishmania braziliensis & LbrM.24.1350 & 170 & 31.84 & 52.51 \\
\hline \multirow[t]{2}{*}{ Leishmania mexicana } & LmxM.24.1500a & 170 & 33.15 & 50.54 \\
\hline & LmxM.24.1500 & 276 & 22.34 & 34.04 \\
\hline Average & & 177.70 & 30.90 & 49.08 \\
\hline
\end{tabular}

${ }^{\mathrm{a}}$ Access number in the UniProt Knowledgebase

${ }^{b}$ Number of amino acids

${ }^{\mathrm{c}} \mathrm{I}$ (Identity) values expressed in percentage (\%)

${ }^{\mathrm{d}} \mathrm{S}$ (Similarity) values expressed in percentage (\%)

maximum likelihood placed it evolutionarily close to the phylum Amoebozoa, particularly of A. castellanii orthologue ACA1_069360, while the algorithm of minimum evolution placed it close to $T$. vaginalis orthologue.

\section{Structural Analysis}

We constructed 3D models of one of the TCTP orthologs in each organism using the human protein as a point of comparison (Fig. 3). First, we realized that the number and arrangement of $\beta$-sheets and $\alpha$-helices is maintained in all species, only with variations in the size of these secondary structures. The tertiary structure was also similar in all the predicted proteins, the main difference being the size of the bridge between the fifth and sixth $\beta$ sheet (flexible loop/ TCTP1), which tends to be larger in the parasites studied compared to human protein.

\section{Discussion}

Given the functional relevance of TCTP in eukaryotes and its possible use as a therapeutic target against protozoan parasites, we carried out an analysis of this protein from several aspects. A moderate identity (38.19\%) was found when aligning the sequences of all the orthologues studied and the TCTP1 and TCPT2 fingerprints were identified in all of them. Among the most conserved residues that we found in the protozoan parasites studied was E123 (corresponding to residue 12 of human TCTP), which previous reports showed that it is an important residue for the binding of TCTP to RHEB (RAS homologue enrichment in brain) GTPase to activate TOR (Target of rapamycin) [23]. In contrast, residues S46 and S64 of human TCTP (residues 163 and 181 of our alignment), which are subject to phosphorylation by PLK, are not conserved in parasites and are only found in mammalian TCTPs [24, 25]. Interestingly, the V186 residue of our alignment that was highly conserved in parasites, in humans (residue 69) is changed by a glycine that participates in the binding of TCTP with the PIM-3 (Provirus integrating site Moloney murine leukemia virus 3) kinase [26]. The conservation of K202 (residue 85 of human TCTP), F255 (residue 114 of human TCTP), G260 (residue 137 of human TCTP) and P284 (residue 158 of human TCTP) may be due to their participation in the homodimerization of the protein [27]. For residue D291 (residue 165 of human TCTP), its participation in the binding of TCTP to G proteins has been suggested [15]. In contrast, the residues corresponding to L78 (residue 195 in our alignment) and Q134 (residue 254 in our alignment) of human TCTP, which are also important for the binding of this protein to $G$ proteins [28], were poorly preserved in parasites. L78 was only present in 52\% of the protozoan parasites, particularly in parasites of the Kinetoplastea class, being mainly changed by a tyrosine, whereas Q134 was only present in $48 \%$ and was exchanged for different amino acids. The latter reinforces the theory that parasite TCTPs can act as dominant negative mutants. It has been mentioned previously that Plasmodium TCTP, which is more efficiently incorporated into B cells than its human counterpart [29], induces the release of histamine, but could block the activation of $\mathrm{B}$ cells by not being able to interact with $\mathrm{G}$ proteins and, therefore, the parasite protects itself, to some extent, from the host's immune system [15].

Regarding our phylogenetic analysis, the clusters generated are largely in agreement with the proposed phylogeny of eukaryotes (Fig. 2) [30,31]. The phylogenetic trees 
Fig. 1 Partial alignment of the sequences of TCTP orthologs identified in protozoan parasites. Consensus was obtained on all available sequences.

TCTP1 and TCTP2 fingerprints are identified by red boxes. The calcium (orange) and microtubule (green) binding regions are underlined. Letters with a red background indicate conserved residues. The predicted secondary structure corresponds to human TCTP. $\beta$-sheets are represented as arrows, while $\alpha$-helices are represented as a spiral line

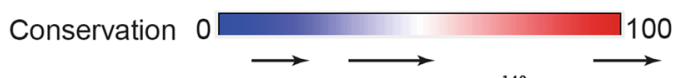

140

160 180

H. sapiens P13693 HDEMFSDIYK IREI---A-A DGL-CLEVEG KMVSRTEGNI DDSLI GGNAS AEG PEGEGTE 63 N. fowleri FDP41_001128 CDVLVTNYYK IEKVF-- -Y GSI--YKVKS HFMIRDPSSF DVSSSPPVGD SEEYHAKH-L 66 N. fowleri NF0012290 GDVLVTNYYK IEKVF-- YY GSI-YKVKS HFMIRDPSSF DVSSSPPVGD SEEYHAKH L 81 T. vaginalis TVAC_180840 EDELVSDAYK LVR--- - - K EDICALEFEG DY I EVDVE-- N. fowleri FDP41_001927 RDELFSDSY- R- - PMKTE-Y EGF-IYNVSS KFMVKDEDED ILYDPDQFEK LENK--C. duodenalis GL50803_6242 GDELLSDA I I TS-- T - T DDL-FYVIKG KMIVADGDEE C. duodenalis DHA2_6242 CDE LLSDAII TS- - - T DDL-FYVIIKG KMIVADGDEE A. castellanii ACA1_069360 DDELASDVY- - - PVEL-E DDV-I LKFTT KLITRTEGNY DMA--GDGGG EETY--D-D 55 L. mexicana LmXM.24.1500a CSEVVCDN-- DCPFGV-E GDI-MYVVGG RYIDVGGEDY GISAN VDEDA AEGATCEV-A 62 L. mexicana LmXM.24.1500 GSEVVCDN-E DCPFGV-E GDI-MYVVGG RYIDVGGEDY GISANVDEDA AEGATCEV-A 168 L. infantum LINF_240020500 GSEVVCDN-- DCPFDV-D GDI-MYVVNG RYIDVGGEDY G I SANVDEDA AEGATCEV-A 62 L. infantum LINF_240020600 GSEVVCDN-E -DCPFDV--E GDI-MYVVNG RYIDVGGEDY GISANVDEDA AEGATCEV-A 62 L. donovani LdBPK_241560.1 CSEVVCDN- - DCPFDV-E GDI-MYVVNG RYIDVGGEDY CISANVDEDA AECATCEV A 62 L. major LmjF.24.1510 GSEVVCDN-- -DCPFDV--E GDI-MYVVNG RYIDVGGEDY GI SANVDEDA AEGATCEV-A 62 L. major LmjF.24.1500 CSEVVCDN-- DCPFDV-E GDI-MYVVNG RYIDVGGEDY CI SANVDEDA AEGATCEV-A 62 T. cruzi TCCLB.506207.50 NAEVVCDN-E DRPMDV E GEI VYVVKG SYIEVGGEDY GIAAN VDEDA GEGAKCEV D 62 T. brucei Tb927.8.6750 NAEVVCDN-- DKPMDL-L DE I-VYAVQG RYIIE IGGEDY GI SANADEDA GEGAACDV-D 62 T. brucei Tb927.8.6760 NAEVVCDN-- -DKPMDV--L DEI-VYAVQG RYIEIGGEDY CISANADEDA AEGAACDV-A 62 A. castellanii ACA1_030760 GDEMFSICY - PFTV - V DDI-VFEVEG KLVSAGGA-V DVDVCCGDAF GGGGDAEQLA 66 E. histolytica EHI_021360 DDEMFSDSFN PT-----VV EGC-MYVVKA KFVVKKEEDF GIACNADEDA EEGAACEACE 61 C. parvum CPATCC_001190 CDEVMSDSYK QTSPFGKAEF DDI-AFEVQS KRVQKSAEDF GIAHNTEEGE AEVV----D 63 C. hominis Chro.30228 GDEVMSDSYK QTSPFGKAEF DDI-AFEVQS KRVQKSAEDF GIAHNTEEGE AEVV-- D 63 P. falciparum PF3D7_0511000 NDEVCSDSYV DQDPFEVPEF REI-AFEVKS NKR IKGNEDY G I ADN S-EDA VEG M---- G 62

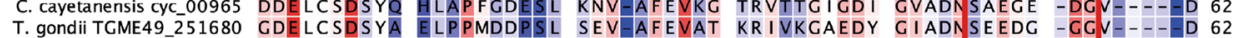
S. neurona SN3_01800135 GDELCSDSYÑ QEAPMDDEDL KDV-AFEVKT RRVVKGAEDY GIADNSEEDG -GGV----D 62 Consensus GDEVVSDNY - -DCPFDV--E GDI-MYVVKG XYIDVGGEDY GISANVDEDA AEGA-G---X Conservation عथع -



H. sapiens P13693 STVITCVDIV MNHHLQETSF TK-FEAYKKY IKDYMKSIKG KLEEQR-PER VKPFMTCAAE 120 N. fowleri FDP41_001128 TNKVLRYSVV EKFGYHECTL KK- KEFLMM MNVYIGTLKS LVEQSGDEER EKSFLMGSTL 124 N. fowleri NF0012290 TNKVLRYSVV EKFGYHECTL KK- - KEFLMM MNVYICTLKS LVEQSCDEER EKSFLMCSTL 139 T. vaginalis TVAC_180840 TRKDTVMN I P YNNNLQKLEL TK-AQFGAW CKKFIPARKA QLSCDA-QTE - -FMQNAKK 99

N. fowleri FDP41_001927 ENTTKVDQIV DSFHLVE IPG IEKVKQLVDT IGPYLSTLRA KIEKES-AEK LAKFNEQVKK 117

N. fowleri NF0009160 ENTTKVDQIV DSFHLVEIPG IEKVKQLVDT IGPYLSTLRA K IEKES AEK LAKFNEQVKK 117

C. duodenalis GL50803_6242 EDAEKVVDVV FRYNLAEAEF TK KTLMSN LKKFMKAMSK NLKKTKSEEE VAAWQEKVNA 100 G. duodenalis DHA2_6242 EDAEKVVDVV FRYNLAEAEF TK- KTLMSN LKKFMKAMSK NLKKTKSEEE VAAWQEKVNA 100 A. castellanii ACA1_069360 STSTTVNNLV DAHKLIATDF TK- KSYMTH IKDYMARLLK KLEAEN-PSR VPAFKKCAQC 112 L. braziliensis LbrM.24.1350 EGKERVVDVV HNNRYTETSY DK-ASYMAH IRGYMKQLLE KIEDEA-EKK A-FQANAAA 117 L. mexicana LmXM.24.1500a EGKQRVVDVV YNNRYTETSY DK-ASYMAH IRSYMKQLLE RIENEE-EKK A-FFTNAAA 117 L. mexicana LmXM.24.1500 EGKQRVVDVV YNNRYTETSY DK- ASYMAH IRSYMKQLLE RIENEE EKK A-FQTNAAA 223 L. infantum LINF_240020500 EGKERVVDVV YNNRYTETSY DK-ASYMAH IRSYMKQLLE KIENEE-ERK V -FQTNAAA 117 L. infantum LINF_240020600 EGKERVVDVV YNNRYTETSY DK ASYMAH IRSYMKQLLE KIENEE ERK V FQTNAAA 117 L. donovani LdBPK_241560.1 EGKERVVDVV YNNRYTETSY DK-ASYMAH IRSYMKQLLE KIENEE-ERK V-FETNAAA 117 L. major LmjF.24.1510 EGKERVVDVV YNNRYTETSY DK-ASYMAH IRSYMKQLLE K IENEE-ERK A-FOTNAAA 117 L. major LmjF.24.1500 EGKERVVDVV YNNRYTETSY DK - ASYMAH IRSYMKQLLE K IENEE ERK A T. cruzi TCCLB.506207.50 DSRQRVVDVV HNNRYTETSY DK- NSYMAH IRGYMKQLLE R IEDEE-EKK K- -FQANAAA 117 T. brucei Tb927.8.6750 DGKQRVIDVV HNNRYTETNY DK- CSYMAH IRGYMKQLLE KIEDEG-AKK A-FQTNAAA 117 T. brucei Tb927.8.6760 DEKQRVIDVV HNNRYTETNY DK NSYMAH IRGYMKQLLE KIEDEG AKK A FQTNAAA 117 A. castellanii ACA1_030760 DAAETV INVV YSHRLVETSF AK- SDFIKY IKAYMNRVKG YLRKTN-PER VAPFMKSAEP 123 E. histolytica EHI_021360 ANVEKVIDVV DNAHLVEQAF TK- TEYMAH IKGYMKKMAD YLQQNH-PEK LDERVATA 118 C. hominis Chro.30228 ADVETVNDII DAFKLESTPF TK- KEYMTY IKAYLAR IKE TMEKSN-PDR VETFMKNAQT 120 P. falciparum PF3D7 0511000 ADVEHVIDIV DSFQLTSTAF SK- KEYSAY IKNYMQKVAK YLEEKK-PDR VEIFKTKAQP 119 C. cayetanensis cyc_00965 DQPETVIDIV DSFHLSETSL SK KDFTAY IKAYIKRVMQ HLEENN PTR VEDFKKGTEA 119

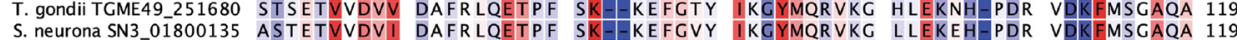
Consensus EGKERVVDVV DNNRLTETSX DK--KSYMAH IKXYMKQLLE KIEKEE-EEK V--FQTNAAA Conservation $\longrightarrow{ }_{260}^{\longrightarrow} \longrightarrow{ }_{280} \longrightarrow$ CQ

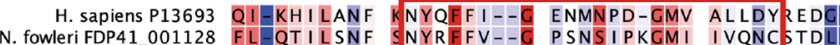

N. fowleri NF0012290 FL-QT I LSNF SNYRFFV--G PSNS I PKGMI IVQNCSTD

T. vaginalis TVAC_180840 FV MWVDANF KEFDFFV-C PTNNVD-DMM LFCKHPESC

N. fowleri FDP41_001927 FLAEQVGKDI KNYKFYQ--G ESNDMDKSML IFQKF NDDC

N. fowleri NFO009160 FLAEQVGKDI KNYKFYQ--C ESNDMDKSML I FOKFNDDC

G. duodenalis GL50803_6242 WV TGLLEKF DDYSFYL-G ASNDVESGMV VFCKWPGA

G. duodenalis DHA2_6242 WV-TGLLEKF DDYSFYL--G ASNDVESGMV VFCKWDGA

A. castellanii ACA1_069360 FV-KKVIADF CEYTFYQ--C EKMDIDNGMI VLSRYSEDC

L. braziliensis LbrM.24.1350 FV-KKVLKDI CDYQFFIPEG NDEDPDNGMI VLCRWDGE

L. mexicana LmXM.24.1500a FV-KKVLKEI DEYQFFIPEG NDEDPDNGMI VLCRWDGE

L. mexicana LmxM.24.1500 FV-KKVLKE I EYQFF I PEG NDEDPDNGM I VLCRWDGE

L. infantum LINF_240020500 FV K KKVLKDI CEYQFF I PEC NDEDPDNGMI VLCRWPGE

L. infantum LINF_240020600 FV-KKVLKDI CEYQFF I PEG NDEDPDNGMI VLCRWDGE

L. donovani LdBPK_241560.1 FV-KKVLKDI CEYQFF I PEG NDEDPDNGMI VLCRWPGE

L. major LmjF.24.1510 FV-KKVLKDI DEYQFF I PEG NDEDPDNGMI VLCRWPGE

NDEDPDNGMI VLCRWPGE

T. cruzi TCCLB.506207.50 FV-KKVI I E I E EQFF I PEC N

T. brucei Tb927.8.6750 FV-KKVIKEI LEYOFFIPEC NEEDPDNGMI VLCKWDCE

T. brucei Tb927.8.6760 FV KKV IKE I E EYQFF I PEG NEEDPDNGM I VLCKWPGE

castellanii ACA1_030760 FV-MKMLKNF [EYSFYM--C ESMDAEAAIG - FGFYKSD

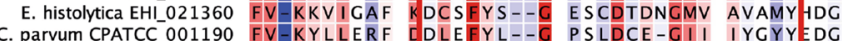

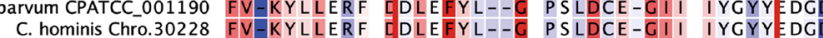

P. falciparum PF3D7_0511000 FI-KHILTNF DDFEFYM- E ESLDMEAC-I IYSYYKGEE

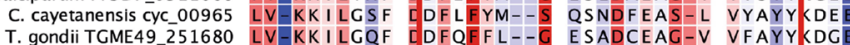

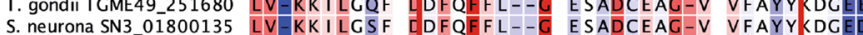

Consensus FV-KKVLKDF DEYQFFT--G NSEDPDNGMI VLCRWDGE -

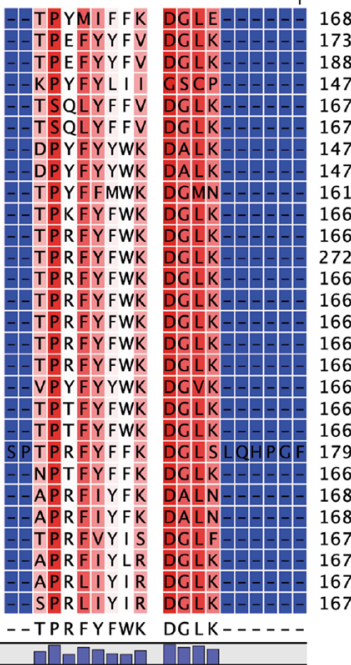




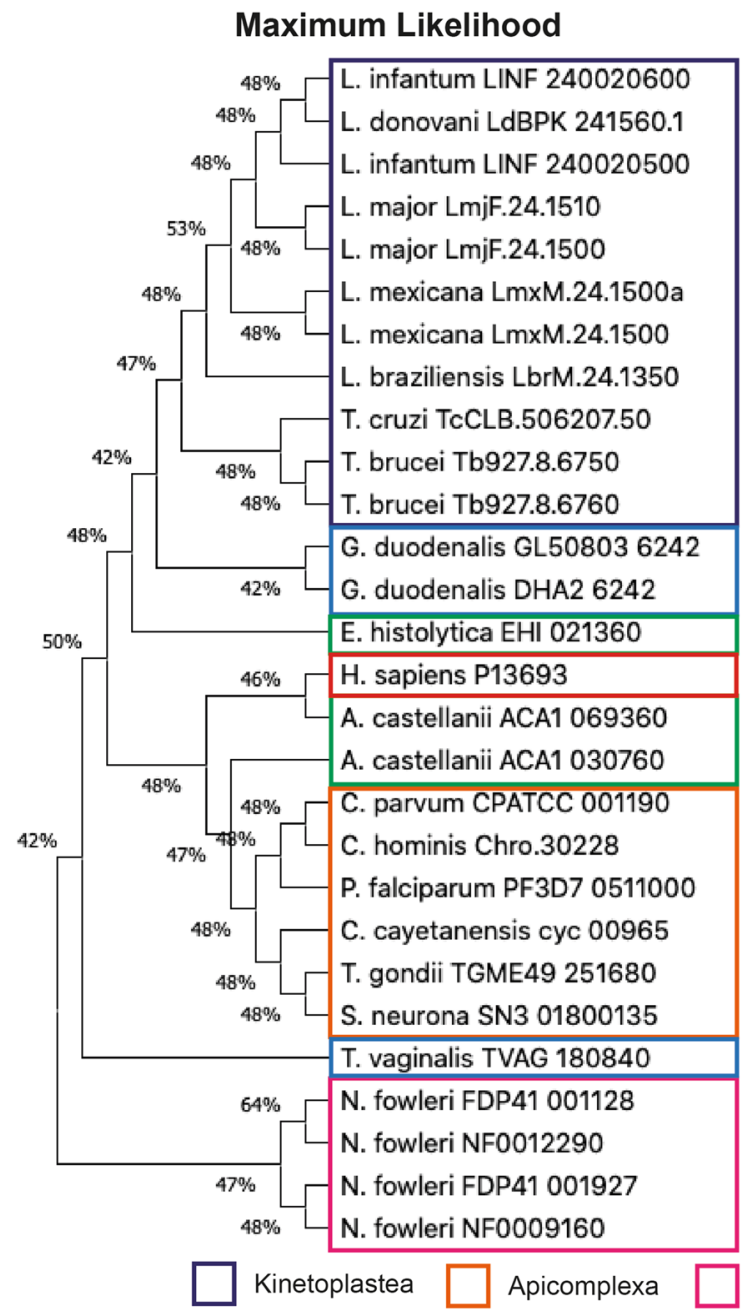

Fig. 2 Phylogenetic trees of TCTP orthologs in protozoan parasites. The evolutionary distances between the species were calculated with the algorithms of maximum likelihood and minimum evolution. The

identified the TCTP orthologs in the amoebo-flagellate $N$. fowleri as the most ancestral proteins. $N$. fowleri belongs to the Heterolobosea clade that diverged from other eukaryotic lineages over a billion years ago [32]. Meanwhile, the phylum Metamonada has also been identified as one of the oldest, and particularly the flagellated protozoan G. duodenalis has been recognized as one of the closest to ancestral eukaryotes, although there is still much debate as to the evidence supporting this claim [33-36]. N. fowleri, T. vaginalis and $G$. duodenalis belong to the Excavata sub-kingdom. The members of the Apicomplexa subphylum belonging to the SAR supergroup (which includes Stramenopiles, Alveolata and Rhizaria) and also the members of the Amoebozoa phylum are considered evolutionarily more advanced that the members of the Excavata sub-kingdom [30, 31]. The evolutionary position of the Kinetoplastea class is not yet well established, but since parasitic kinetoplastids have arisen

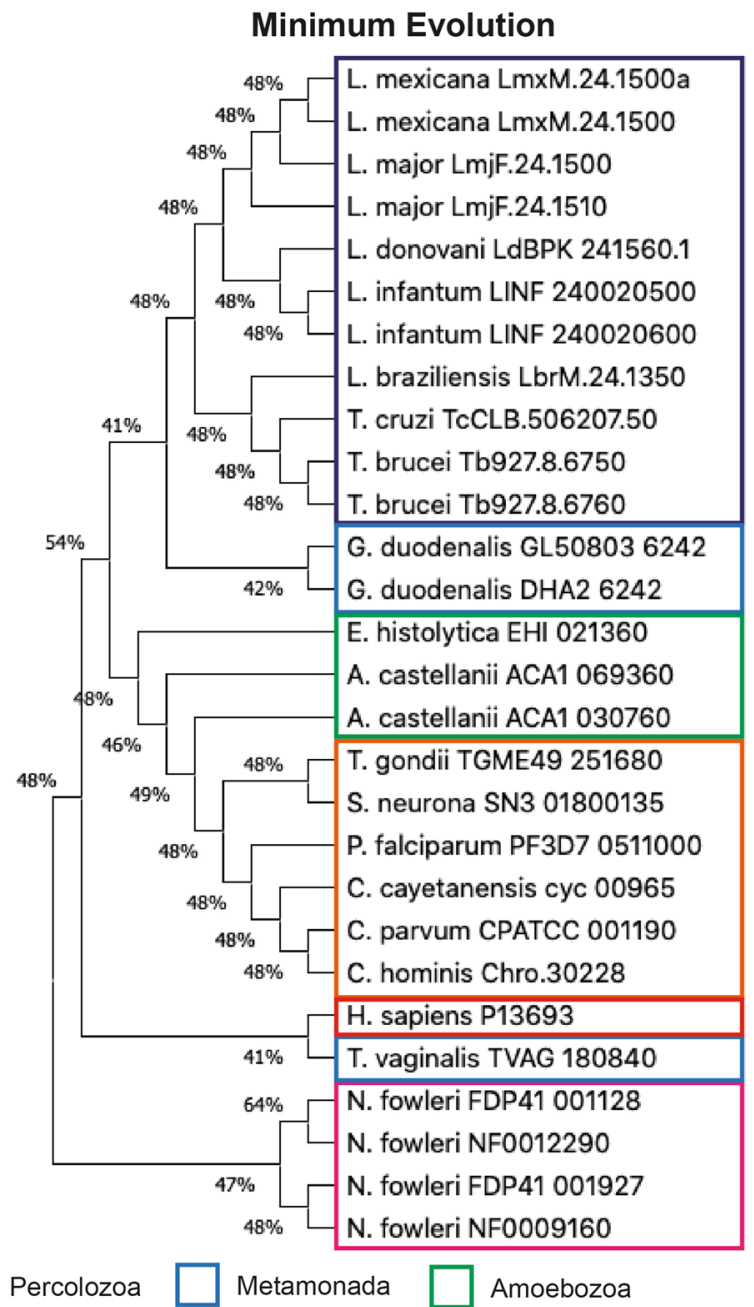

percentage of replicate trees in which the associated taxa clustered together in the bootstrap test are shown next to the branches. The sequence of human TCPT was added for comparative purposes

from non-pathogenic free-living ancestors, multiple processes must have occurred before this step could be taken [37].

Structural analysis of TCTP orthologs predicted a highly conserved secondary and tertiary structure (Fig. 3). Furthermore, an increase in flexible loop size was observed in most TCTP orthologs compared to human protein. Although we do not know the functional reason for this change, it is known that the flexible loop is critical to promote cytokine release and the limitation of the flexibility of this region by disulfide bonds is required [38]. Notably, this area has also served as a binding site for potential TCTP inhibitors (Brompheniramine, Sertraline, ZINC19364226, ZINC19361042, ZINC03831041, ZINC12657067, ZINC12657071, ZINC12863423, ZINC08918508) [39]. This opens up the option of creating new therapeutic strategies against protozoan parasites taking TCTP as a target, which would also 


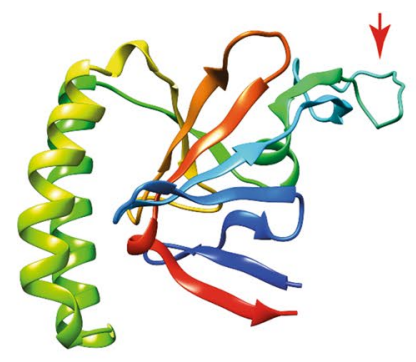

H. sapiens $\mathrm{P} 13693$

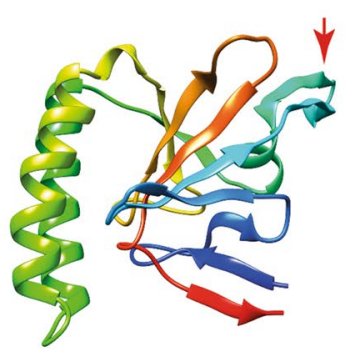

T. vaginalis TVAG_180840

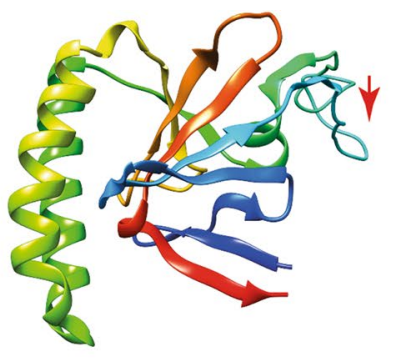

N. fowleri NF0009160
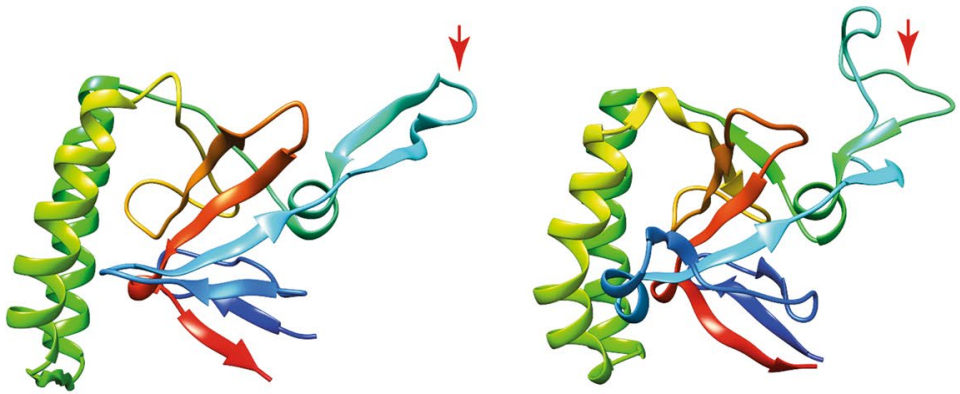

G. duodenalis GL50803_6242

C. cayetanensis cyc_00965

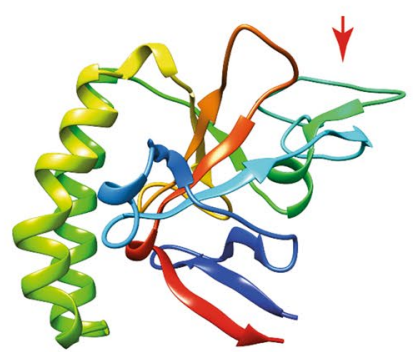

T. gondii TGME49_251680
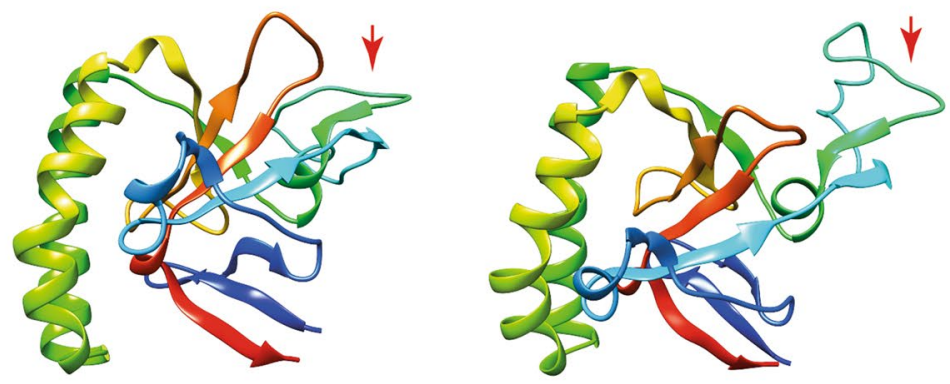

C. parvum CPATCC_001190
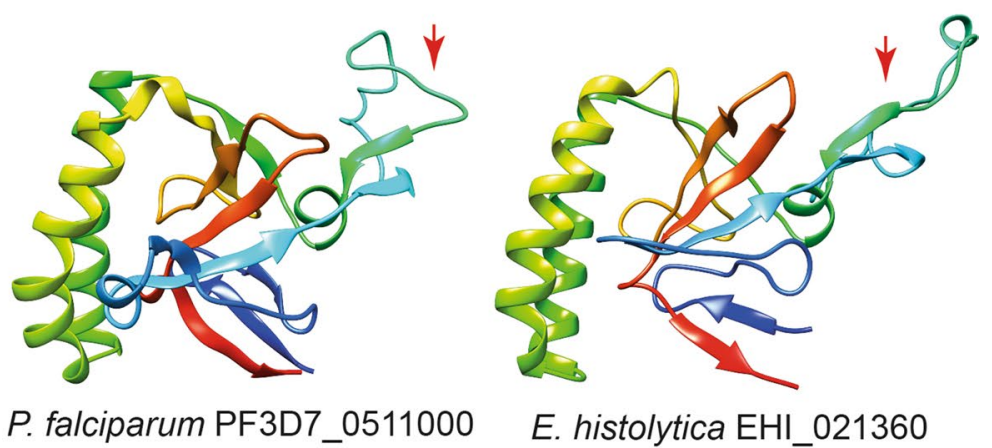

E. histolytica EHI_021360

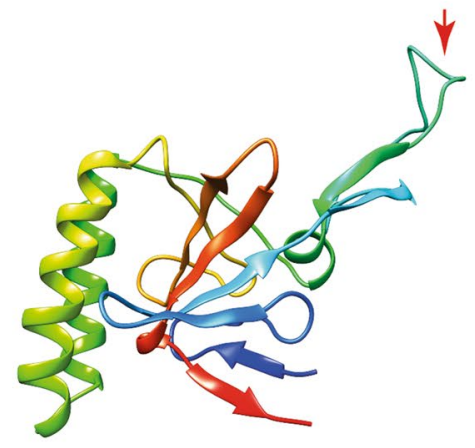

L. infantum LINF_240020600

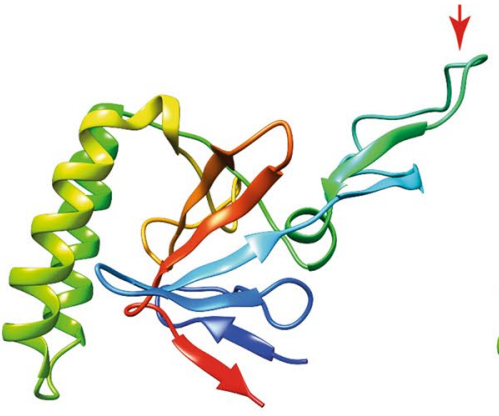

T. cruzi TcCLB.506207.50

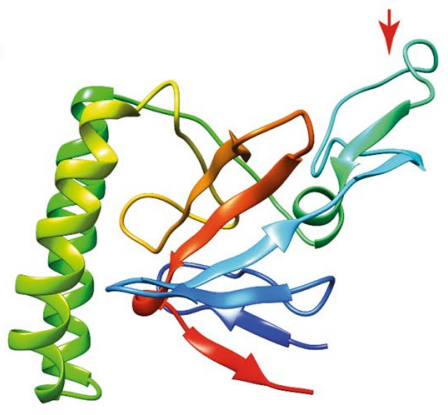

A. castellanii ACA1_069360
Fig. 3 Comparison of predicted three-dimensional structures of TCTP orthologs in protozoan parasites. Structures are represented as ribbons and chains. Note the high level of structural similarity exhib-

take advantage of the differences in this region compared to human protein. P. falciparum TCTP has been suggested to be a potential target for artemisinin, one of the most effective antimalarial drugs, and some residues involved in binding have been described. The $\mathrm{C} 14$ residue (position 115 of ited between these representative TCTPs. The flexible loop, which is the region with the most notable differences between TCTP orthologs studied, is indicated by a red arrow

our alignment) of $P$. falciparum TCTP and other species of the subphylum Apicomplexa has been proposed as one of the possible artemisinin alkylation sites and could serve as an electron donor during endoperoxide activation [40, 41]. However, in species of the Kinetoplastea class, this cysteine 
is found in position 15 (116 of our alignment) and in the other species of protozoan parasites, this residue is replaced by hydrophobic amino acids (phenylalanine, valine, alanine or leucine) (Fig. 1) that have been shown to completely inhibit binding to artemisinin [40]. The F8A mutation (position 119 of our alignment) also significantly decreased the binding of the P. falciparum TCTP protein to artemisinin [40], which is important since in human and most other protozoan parasites, it is replaced by a leucine or isoleucine (Supplementary Fig. 1). D11A or F18A mutations (positions 122 and 129 of our alignment) had only a small effect on artemisinin binding [40]. Furthermore, the regions corresponding to residues 19-46 (130-154 in our alignment), 108-134 (229-255 in our alignment) and 140-163 (265-290 in our alignment) have been reported to be covalently modified by artemisinin radicals [42]. Another thing that has to be considered when designing TCTP inhibitor drugs is that it is a dynamic molecule and in a study where a series of putative compounds inhibiting human TCTP were found [43], the amino acids most involved in the interaction with the chemicals were K34, M35, V36, G69, V70, D71, I72 and P158 (residues K151, M152, V153, G195, V196, D197, I198, P283 in our alignment).

In conclusion, we can say that TCTP orthologues have important functions in protozoan parasites, they maintain a moderate identity between them, but their structure is quite conserved, evolutionarily they follow the proposed phylogeny of eukaryotes and the changes in the flexible loop could be used in the creation of new antiparasitic drugs.

Supplementary Information The online version contains supplementary material available at https://doi.org/10.1007/s11686-022-00521-9.

Funding Open access funding provided by Uppsala University. This research did not receive any specific grant from funding agencies in the public, commercial, or not-for-profit sectors.

\section{Declarations}

Conflict of Interest The author declares no conflict of interest.

Ethical Statement This material is the original work of the author and has not been previously published elsewhere. Bioinformatic work does not require an ethical permit and the goal is to reduce work with animals.

Open Access This article is licensed under a Creative Commons Attribution 4.0 International License, which permits use, sharing, adaptation, distribution and reproduction in any medium or format, as long as you give appropriate credit to the original author(s) and the source, provide a link to the Creative Commons licence, and indicate if changes were made. The images or other third party material in this article are included in the article's Creative Commons licence, unless indicated otherwise in a credit line to the material. If material is not included in the article's Creative Commons licence and your intended use is not permitted by statutory regulation or exceeds the permitted use, you will need to obtain permission directly from the copyright holder. To view a copy of this licence, visit http://creativecommons.org/licenses/by/4.0/.

\section{References}

1. Xoconostle-Cázares B, Ruiz-Medrano R (2017) Structure-function relationship of TCTP. In: Telerman A, Amson R (eds) TCTP/ tpt1—remodeling signaling from stem cell to disease. Springer International Publishing, Cham, pp 47-68

2. Bommer U-A, Telerman A (2020) Dysregulation of TCTP in biological processes and diseases. Cells 9:1632. https://doi.org/10. 3390/cells9071632

3. Zheng J, Chen Y, Li Z et al (2018) Translationally controlled tumor protein is required for the fast growth of Toxoplasma gondii and maintenance of its intracellular development. FASEB J 32:906-919. https://doi.org/10.1096/fj.201700994R

4. Meyvis Y, Houthoofd W, Visser A et al (2009) Analysis of the translationally controlled tumour protein in the nematodes Ostertagia ostertagi and Caenorhabditis elegans suggests a pivotal role in egg production. Int J Parasitol 39:1205-1213. https:// doi.org/10.1016/j.ijpara.2009.02.017

5. Gnanasekar M, Rao KVN, Chen L et al (2002) Molecular characterization of a calcium binding translationally controlled tumor protein homologue from the filarial parasites Brugia malayi and Wuchereria bancrofti. Mol Biochem Parasitol 121:107-118. https://doi.org/10.1016/S0166-6851(02)00027-0

6. Gnanasekar M, Dakshinamoorthy G, Ramaswamy K (2009) Translationally controlled tumor protein is a novel heat shock protein with chaperone-like activity. Biochem Biophys Res Commun 386:333-337. https://doi.org/10.1016/j.bbrc.2009.06.028

7. Mak CH, Sun KW, Ko RC (2001) Identification of some heatinduced genes of Trichinella spiralis. Parasitology 123:293-300. https://doi.org/10.1017/S0031182001008320

8. Gnanasekar M, Ramaswamy K (2007) Translationally controlled tumor protein of Brugia malayi functions as an antioxidant protein. Parasitol Res 101:1533-1540. https://doi.org/10.1007/ s00436-007-0671-z

9. Bhisutthibhan J, Pan X-Q, Hossler PA et al (1998) The Plasmodium falciparum translationally controlled tumor protein homolog and its reaction with the antimalarial drug artemisinin. J Biol Chem 273:16192-16198. https://doi.org/10.1074/jbc.273.26. 16192

10. Bhisutthibhan J, Meshnick SR (2001) Immunoprecipitation of [3 H]dihydroartemisinin translationally controlled tumor protein (TCTP) adducts from Plasmodium falciparum-infected erythrocytes by using anti-TCTP antibodies. Antimicrob Agents Chemother 45:2397-2399. https://doi.org/10.1128/AAC.45.8.23972399.2001

11. Afonso A, Hunt P, Cheesman S et al (2006) Malaria parasites can develop stable resistance to artemisinin but lack mutations in candidate genes atp6 (encoding the sarcoplasmic and endoplasmic reticulum $\mathrm{Ca} 2+$ ATPase), tctp, mdr1, and cg10. Antimicrob Agents Chemother 50:480-489. https://doi.org/10.1128/AAC. 50.2.480-489.2006

12. MacDonald SM, Bhisutthibhan J, Shapiro TA et al (2001) Immune mimicry in malaria: Plasmodium falciparum secretes a functional histamine-releasing factor homolog in vitro and in vivo. Proc Natl Acad Sci 98:10829-10832. https://doi.org/10.1073/pnas.20119 1498

13. Bossard G, Rodrigues V, Tour E, Geiger A (2021) Mice immunization with Trypanosoma brucei gambiense translationally controlled tumor protein modulates immunoglobulin and cytokine production, as well as parasitaemia and mice survival after 
challenge with the parasite. Infect Genet Evol 87:104636. https:// doi.org/10.1016/j.meegid.2020.104636

14. Rao KVN, Chen L, Gnanasekar M, Ramaswamy K (2002) Cloning and characterization of a calcium-binding, histamine-releasing protein from Schistosoma mansoni. J Biol Chem 277:3120731213. https://doi.org/10.1074/jbc.M204114200

15. Hinojosa-Moya J, Xoconostle-Cázares B, Piedra-Ibarra E et al (2008) Phylogenetic and structural analysis of translationally controlled tumor proteins. J Mol Evol 66:472-483. https://doi.org/10. 1007/s00239-008-9099-z

16. Taylor KJ, Van TTH, MacDonald SM et al (2015) Immunization of mice with Plasmodium TCTP delays establishment of Plasmodium infection. Parasite Immunol 37:23-31. https://doi.org/10. 1111/pim.12158

17. Pelleau S, Diop S, Dia Badiane M et al (2012) Enhanced basophil reactivities during severe malaria and their relationship with the Plasmodium falciparum histamine-releasing factor translationally controlled tumor protein. Infect Immun 80:2963-2970. https://doi. org/10.1128/IAI.00072-12

18. Aurrecoechea C, Barreto A, Basenko EY et al (2017) EuPathDB: the eukaryotic pathogen genomics database resource. Nucleic Acids Res 45:D581-D591. https://doi.org/10.1093/nar/gkw1105

19. Blum M, Chang H-Y, Chuguransky S et al (2021) The InterPro protein families and domains database: 20 years on. Nucleic Acids Res 49:D344-D354. https://doi.org/10.1093/nar/gkaa977

20. Tamura K, Stecher G, Kumar S (2021) MEGA11: molecular evolutionary genetics analysis version 11. Mol Biol Evol 38:30223027. https://doi.org/10.1093/molbev/msab120

21. Biasini M, Bienert S, Waterhouse A et al (2014) SWISS-MODEL: modelling protein tertiary and quaternary structure using evolutionary information. Nucleic Acids Res 42:W252-W258. https:// doi.org/10.1093/nar/gku340

22. Pettersen EF, Goddard TD, Huang CC et al (2004) UCSF Chimera - a visualization system for exploratory research and analysis. J Comput Chem 25:1605-1612. https://doi.org/10.1002/jcc. 20084

23. Kumar R, Maurya R, Saran S (2020) Investigating the role of translationally control tumor protein in growth, development and differentiation of Dictyostelium discoideum. Front Cell Dev Biol 8:1-13. https://doi.org/10.3389/fcell.2020.00742

24. Yarm FR (2002) Plk phosphorylation regulates the microtubulestabilizing protein TCTP. Mol Cell Biol 22:6209-6221. https:// doi.org/10.1128/MCB.22.17.6209-6221.2002

25. Cucchi U, Gianellini LM, De Ponti A et al (2010) Phosphorylation of TCTP as a marker for polo-like kinase-1 activity in vivo. Anticancer Res 30:4973-4985

26. Zhang F, Liu B, Wang Z et al (2013) A novel regulatory mechanism of Pim-3 kinase stability and its involvement in pancreatic cancer progression. Mol Cancer Res 11:1508-1520. https://doi. org/10.1158/1541-7786.MCR-13-0389

27. Malard F, Assrir N, Alami M et al (2018) Conformational ensemble and biological role of the TCTP intrinsically disordered region: influence of calcium and phosphorylation. J Mol Biol 430:1621-1639. https://doi.org/10.1016/j.jmb.2018.04.024

28. Hsu Y-C, Chern JJ, Cai Y et al (2007) Drosophila TCTP is essential for growth and proliferation through regulation of $\mathrm{dRheb}$ GTPase. Nature 445:785-788. https://doi.org/10.1038/natur e05528

29. Calderón-Pérez B, Xoconostle-Cázares B, Lira-Carmona R et al (2014) The Plasmodium falciparum translationally controlled tumor protein (TCTP) is incorporated more efficiently into B cells than its human homologue. PLoS ONE 9:e85514. https://doi.org/ 10.1371/journal.pone.0085514

30. Adl SM, Simpson AGB, Farmer MA et al (2005) The new higher level classification of eukaryotes with emphasis on the taxonomy of protists. J Eukaryot Microbiol 52:399-451. https://doi.org/10. 1111/j.1550-7408.2005.00053.x

31. Adl SM, Simpson AGB, Lane CE et al (2012) The revised classification of eukaryotes. J Eukaryot Microbiol 59:429-514. https:// doi.org/10.1111/j.1550-7408.2012.00644.x

32. Fritz-Laylin LK, Prochnik SE, Ginger ML et al (2010) The genome of Naegleria gruberi illuminates early eukaryotic versatility. Cell 140:631-642. https://doi.org/10.1016/j.cell.2010.01. 032

33. Lloyd D, Harris JC (2002) Giardia: highly evolved parasite or early branching eukaryote? Trends Microbiol 10:122-127. https:// doi.org/10.1016/S0966-842X(02)02306-5

34. He D, Fiz-Palacios O, Fu C-J et al (2014) An alternative root for the eukaryote tree of life. Curr Biol 24:465-470. https://doi.org/ 10.1016/j.cub.2014.01.036

35. Lagunas-Rangel FA, Bermúdez-Cruz RM (2019) Epigenetics in the early divergent eukaryotic Giardia duodenalis: an update. Biochimie 156:123-128. https://doi.org/10.1016/j.biochi.2018.10.008

36. Lagunas-Rangel FA, Yee J, Bermúdez-Cruz RM (2021) An update on cell division of Giardia duodenalis trophozoites. Microbiol Res 250:126807. https://doi.org/10.1016/j.micres.2021.126807

37. Cenci U, Moog D, Curtis BA et al (2016) Heme pathway evolution in kinetoplastid protists. BMC Evol Biol 16:109. https://doi.org/ 10.1186/s12862-016-0664-6

38. Lee H, Kim M-S, Lee J-S et al (2020) Flexible loop and helix 2 domains of TCTP are the functional domains of dimerized TCTP. Sci Rep 10:197. https://doi.org/10.1038/s41598-019-57064-9

39. Kumar R, Maurya R, Saran S (2017) Identification of novel inhibitors of the translationally controlled tumor protein (TCTP): insights from molecular dynamics. Mol Biosyst 13:510-524. https://doi.org/10.1039/C6MB00850J

40. Li W, Zhou Y, Tang G, Xiao Y (2016) Characterization of the artemisinin binding site for translationally controlled tumor protein (TCTP) by bioorthogonal click chemistry. Bioconjug Chem 27:2828-2833. https://doi.org/10.1021/acs.bioconjchem.6b00556

41. Chae J, Choi I, Kim C (2006) Homology modeling and molecular docking study of translationally controlled tumor protein and artemisinin. Arch Pharm Res 29:50-58. https://doi.org/10.1007/ BF02977468

42. Eichhorn T, Winter D, Büchele B et al (2013) Molecular interaction of artemisinin with translationally controlled tumor protein (TCTP) of Plasmodium falciparum. Biochem Pharmacol 85:3845. https://doi.org/10.1016/j.bcp.2012.10.006

43. Fischer N, Seo E-J, Klinger A et al (2021) AMG900 as novel inhibitor of the translationally controlled tumor protein. Chem Biol Interact 334:109349. https://doi.org/10.1016/j.cbi.2020. 109349

Publisher's Note Springer Nature remains neutral with regard to jurisdictional claims in published maps and institutional affiliations. 